\title{
Tratamento cirúrgico por via anterior na mielopatia cervical espondilótica com seguimento mínimo de dez anos
}

\author{
Anterior decompression and fusion for spondilotic cervical \\ mielopathy with a minimal ten-year follow-up \\ Tratamiento quirúrgico por vía anterior en la mieolopatía cervical \\ espondilótica con seguimiento mínimo de diez años
}

\author{
Rui Peixoto Pinto' \\ José Oliveira' ${ }^{1}$ \\ Rui Matos ${ }^{1}$ \\ Nuno Neves' \\ Manuel Ribeiro da Silva' \\ Pedro Cacho Rodrigues ${ }^{1}$ \\ Rui M Barros ${ }^{2}$ \\ Abel Trigo Cabral'
}

\section{RESUMO}

Objetivo: a mielopatia cervical espondilótica (MEC) é uma causa frequente de disfunção da medula espinhal na população adulta. $\mathrm{O}$ tratamento implica em descompressão cirúrgica precoce. O objetivo foi apresentar um estudo retrospectivo da descompressão anterior e artrodese para MEC com um seguimento mínimo de dez anos. Métodos: pacientes operados entre Janeiro de 1990 e Dezembro de 1994 foram avaliados por sexo, idade, número de níveis operados, avaliação funcional pela escala de Nurick préoperatória um ano após cirurgia e após a revisão final que ocorreu em 2004, evidência de consolidação e complicações. Resultados: foram avaliados 91 pacientes, 69 do sexo masculino, 22 do sexo feminino, com uma média de idade de 56,6 anos (42-86) e um seguimento médio de 11,9 anos. Ocorreram cinco óbitos: três pacientes no pós-operatório imediato, um no

\begin{abstract}
Objective: cervical spondylotic myelopathy (CSM) is a common cause of spinal cord dysfunction in the adult population. The treatment implies early surgical decompression. The objective was to present a retrospective study of anterior decompression and arthrodesis for CSM with a minimal ten years follow-up. Methods: patients operated between January 1990 and December 1994 were evaluated for sex, age, number of levels operated, functional evaluation with Nurick scale preoperatively, one year after surgery and at the final revision that took place in 2004, evidence of consolidation and complications. Results: 91 patients were evaluated, 69 male, 22 female, with a mean age of 56.6 years (42-86) and mean follow-up of 11.9 years. Five deaths occurred: three patients died in the immediate postoperative period, one in the first year and one during the

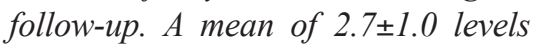

\section{RESUMEN}

Objetivo: la mielopatía cervical espondilótica (MEC) es una causa frecuente de disfunción de la médula espinal en la población adulta. El tratamiento implica una descompresión quirúrgica precoz. El objetivo es presentar un estudio retrospectivo de la descompresión anterior y artrodesis para MEC con un seguimiento mínimo de diez años. Métodos: pacientes operados entre Enero de 1990 y Diciembre de 1994 fueron evaluados según el sexo, la edad, el número de niveles operados, la evaluación funcional por la escala de Nurick pre operatoria un año después de la cirugía $y$ después de la revisión final que fue en el 2004, evidencia de consolidación y complicaciones. Resultados: fueron evaluados 91 pacientes, 69 del sexo masculino, 22 del sexo femenino, con un promedio de edades de 56.6 años (42 a 86) y un seguimiento promedio de 11.9 años. Ocurrieron cinco óbitos: tres pacientes en el postoperatorio in-

\footnotetext{
Serviço de Ortopedia do Hospital de São João - HS

Faculdade de Medicina da Universidade do Porto - FMUP - Porto, Portugal.

'Grupo da Coluna do Serviço de Ortopedia do Hospital de São João - HSJ - Faculdade de Medicina da Universidade do Porto - FMUP - Porto, Portugal.

${ }^{2}$ Biostrument S.A. - Porto, Portugal.
} 
primeiro ano e um durante o restante período. Em média, foram operados $2,7 \pm 1,0$ níveis por paciente (1-4). O valor médio de Nurick pré-operatório foi de $3,8 \pm 0,9$. Houve uma melhoria significativa do estado neurológico um ano após a cirurgia $(2,2 \pm 1,1 ; p<0,001)$ e entre o pré-operatório e a avaliação final $(2,3 \pm 1,2 ; p<0,001)$. A degradação entre o primeiro ano e a avaliação final foi estatisticamente significativa $(p=0,004)$. Verificou-se uma forte correlação entre a idade e o número de níveis operados $(\mathrm{r}=0,391 ; \mathrm{p}=0,01)$, idade e estado neurológico inicial $(\mathrm{r}=0,238$; $\mathrm{p}=0,05)$, estado neurológico inicial e número de níveis operados $(\mathrm{r}=0,251$; $\mathrm{p}=0,05)$ e sexo e número de níveis operados, sendo as mulheres operadas a mais níveis $(\mathrm{r}=0,208 ; \mathrm{p}=0,05)$. Verificou-se também uma maior deterioração neurológica entre o primeiro ano e a avaliação final em doentes mais jovens em comparação com os idosos $(\mathrm{r}=0,25 ; \mathrm{p}=0,05)$. Há uma forte correlação entre a recuperação obtida ao primeiro ano e a recuperação final $(\mathrm{r}=0,838 ; \mathrm{p}=0,01)$. A taxa de consolidação foi de $100 \%$. Conclusão: na MEC, a cirurgia proporciona uma melhoria neurológica significativa e, apesar de uma deterioração clínica entre o primeiro ano e a avaliação final, os benefícios da descompressão são evidentes pelo menos dez anos após a intervenção.

DESCRITORES: Estenose espinal; Doenças da medula espinal; Descompressão cirúrgica; Resultado de tratamento for patient was performed (1-4). The mean value of preoperative Nurick was 3.8 \pm 0 .9. There was a significant improvement in neurological condition after one year $(2.2 \pm 1.1 ; p<0.001)$, and between preoperative period and final evaluation $(2.3 \pm 1.2 ; p<0.001)$. The degradation between the first year and the final evaluation was statistically significant $(p=0.004)$. There was a strong correlation between age and the number of operated levels $(r=0.391 ; p=0.01)$, age and initial neurologic status $(r=0.238 ; p=0.05)$, initial neurological status and number of operated levels $(r=0.251 ; p=0.05$ ) and sex and number of operated levels, with women being operated for more levels $(r=0.208 ; p=0.05)$. There was also a stronger neurological deterioration between the first year and the final follow-up in young patients when compared to older ones ( $r=0.25$; $p=0.05)$. There is a strong relation between the first year improvement and the final improvement $(r=0.838$, $p=0.01$ ). There was a $100 \%$ rate of consolidation. Conclusion: in CSM, a significant neurological improvement can be expected with surgery, and despite a clinical deterioration between the first year and the final evaluation, the benefits of decompression are evident at least ten years after the intervention.

KEYWORDS: Spinal stenosis; Spinal cord diseases; Decompression, surgical; Treatment outcome mediato, uno durante el primer año y uno durante el restante periodo. En promedio, fueron operados $2.7 \pm 1.0$ niveles por paciente (1 a 4). El valor promedio de Nurick pre operatorio fue de 3.8 0 0.9. Hubo una mejora significativa del estado neurológico un año después de la cirugía (2.2 $\pm 1.1 ; p<0.001)$, y entre el pre operatorio y la evaluación final (2.3 $\pm 1.2 ; p<0.001)$. El agravamiento entre el primer año y la evaluación final fue estadísticamente significativa $(p=0.004)$. Se verificó una correlación fuerte entre la edad y el número de niveles operados ( $r=0.391 ; p=0.01)$, edad $y$ estado neurológico inicial $(r=0.238$; $p=0.05)$, estado neurológico inicial y número de niveles operados ( $r=0.251$; $p=0.05)$, y sexo y número de niveles operados, siendo las mujeres operadas con más niveles $(r=0.208 ; p=0.05)$. Se verificó también un mayor deterioro neurológico entre el primer año y la evaluación final en enfermos más jóvenes al ser comparados con pacientes de edad avanzada $(r=0.250 ; p=0.05)$. Hay una fuerte correlación entre la recuperación obtenida en el primer año y la recuperación final ( $r=0.838 ; p=0.01$ ). La tasa de consolidación fue del 100\%. Conclusión: en la MEC, la cirugía proporciona una mejora neurológica significativa y, a pesar de un deterioro clínico entre el primer año y la evaluación final, los beneficios de la descompresión son evidentes por lo menos diez años después de la intervención.

DESCRIPTORES: Estenosis espinal; Enfermedades de la médula espinal; Descompresión quirúrgica; Resultado del tratamiento

\section{INTRODUÇÃO}

A mielopatia cervical espondilótica é a causa mais frequente de disfunção da medula espinhal na população adulta. Os doentes afetados representam uma grande parte dos indivíduos que são sujeitos a intervenção cirúrgica à coluna cervical por condições degenerativas.

Os subtis achados clínicos que constituem as manifestações primárias da mielopatia cervical espondilótica são de difícil diagnóstico, e verdadeiros estudos sobre a história natural dessa patologia são inexistentes. Os pacientes ou seus familiares notam alterações progressivas na marcha ou no equilíbrio e, muitas vezes, atribuem essas alterações a fenómenos de degeneração osteoarticular nas articulações dos membros inferiores ${ }^{1}$.

O tratamento cirúrgico é considerado uma opção válida na maior parte dos doentes com um quadro de mielopatia cervical definido, numa tentativa de reverter ou atrasar o processo de degeneração neurológica. No entanto, ainda 
não foi possível definir um algoritmo de tratamento universal, dada a variabilidade do quadro de apresentação inicial e a forma como a doença se desenvolve nos diferentes doentes, bem como devido à falta de estudos prospectivos e randomizados que estratifiquem as diferentes opções de tratamento em função dos distintos quadros de apresentação da doençâa .

$\mathrm{O}$ tratamento cirúrgico dessa doença degenerativa cervical evoluiu nos últimos 70 anos. Mixter e Barr descreveram, em 1934, uma forma de abordagem posterior, ao realizarem as primeiras laminectomias cervicais. Nos anos 1970, várias técnicas de laminoplastia foram introduzidas como uma alternativa mais estabilizadora à laminectomia ${ }^{3,4}$

Nos anos 1950, ganharam popularidade procedimentos por abordagem anterior, com Robinson e $\mathrm{Smith}^{5}$, Cloward ${ }^{6}$ e Simmons e Bhalla ${ }^{7}$, descrevendo formas de descompressão anterior e técnicas de fusão envolvendo o uso de enxerto ósseo de crista ilíaca. Um aumento da familiaridade com a via de abordagem anterior, bem como aparentes bons resultados clínicos, com evolução neurológica positiva e favorável logo nos primeiros tempos pós-operatórios, levou a um aumento na frequência de utilização dessa via, bem como ao desenvolvimento de técnicas de descompressão e estabilização multilevel. Os objetivos do tratamento cirúrgico, independentemente da técnica utilizada, são prevenir a deterioração neurológica, ou até mesmo reverter, ainda que parcialmente, o quadro de agravamento neurológico. Os princípios subjacentes a esses objetivos são a descompressão apropriada da medula, restaurando uma morfologia adequada, permitindo uma diminuição do edema medular e com provável melhora da circulação sanguínea, influindo na recuperação neurológica. A estabilização da coluna cervical é considerada nos casos em que a mobilidade segmentar é considerada um fator de agravamento clínico e para prevenir o desenvolvimento de deformidades tardias que prejudiquem o resultado cirúrgico.

O objetivo deste trabalho foi avaliar os resultados funcionais da descompressão e artrodese anterior no tratamento da mielopatia cervical espondilótica e sua evolução a longo prazo, bem como os fatores que influenciam esses resultados.

\section{MÉTODOS}

Foram analisados os processos clínicos de pacientes sofrendo de mielopatia cervical espondilótica submetidos a descompressão e artrodese anterior, entre Janeiro de 1990 e Dezembro de 1994, tendo sido registados o sexo, idade, número de níveis intervencionados, avaliação funcional segundo a escala de Nurick no pré-operatório e no final do primeiro ano, evidência de consolidação e complicações.

Todos os pacientes foram operados pelos autores sénior (JCO e RP), tendo sido utilizada a técnica de Cloward ${ }^{6}$ para compressões a um único nível (Figura 1) e a técnica de Senegas ${ }^{8}$ para dois ou mais níveis, complementada, nesses casos, com placa em batente (Figura 2).

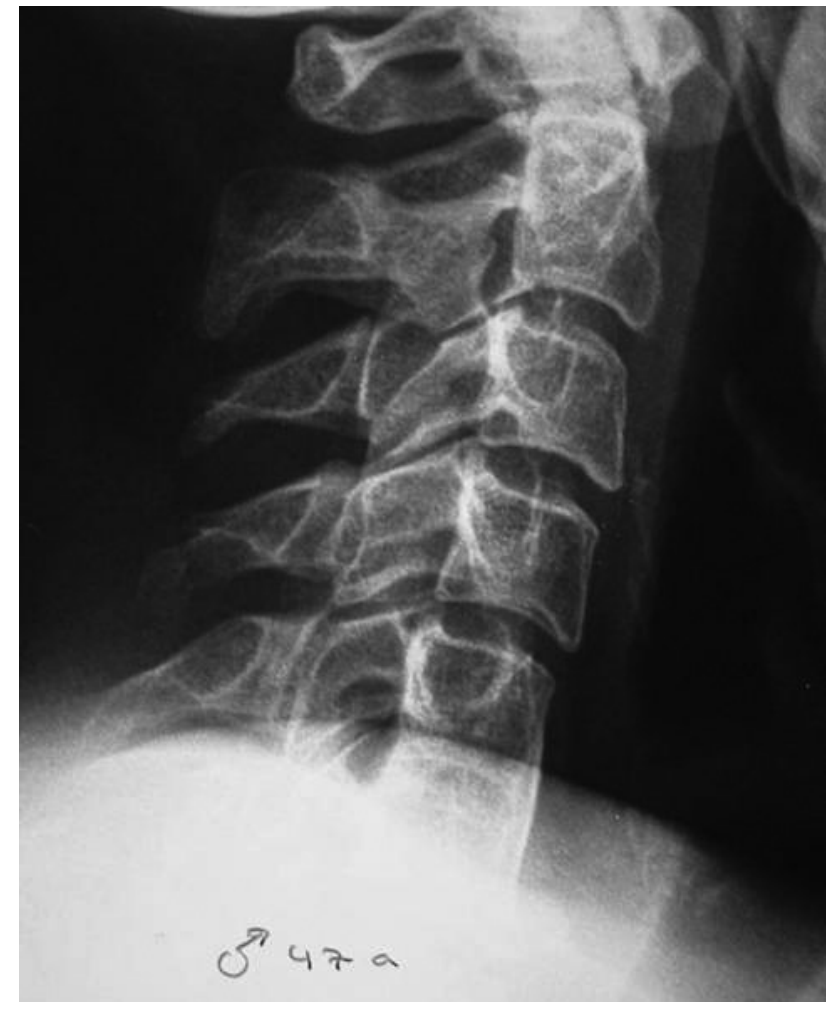

Figura 1

Técnica de Cloward ${ }^{6}$ para compressões a um único nível

Os pacientes foram revisados em 2005 e 2006, altura em que se procedeu a nova avaliação funcional e registo de complicações ocorridas.

A análise estatística envolveu o teste de $t$ de Student emparelhado e análise de correlação, utilizando o programa Statistical Package for Social Sciences (SPSS), versão 16.1.

\section{RESULTADOS}

Foram identificados 91 pacientes, 69 do sexo masculino e 22 do sexo feminino, com uma média de idade de 56,6 anos (42-86) e um tempo médio de seguimento de 11,9 anos.

Foram excluídos cinco pacientes por óbito, três no período pós-operatório imediato $(3,3 \%)$, um no primeiro ano e outro após o primeiro tempo de avaliação. Também foram excluídos 9 processos incompletos e 18 pacientes por não comparecerem à consulta de revisão. No total, foram revisados $80,2 \%$ dos pacientes.

Operados em 1 nível 14 casos, 2 níveis em 21 casos, 3 níveis em 34 casos e 4 níveis em 22 casos, para uma média de $2,7 \pm 1,0$. O estado neurológico, segundo a escala de

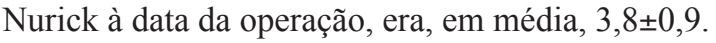

Pelo teste de $t$ de Student emparelhado, verificou-se uma melhoria significativa do estado neurológico entre o pré-operatório e o primeiro ano após a operação $(2,2 \pm 1,1$; $\mathrm{p}<0,001)$ e igualmente entre o pré-operatório e a última revisão $(2,3 \pm 1,2 ; \mathrm{p}<0,001)$. Entre o primeiro ano após a operação e a última revisão, ocorreu uma degradação clínica estatisticamente significativa $(\mathrm{p}=0,004)$. 


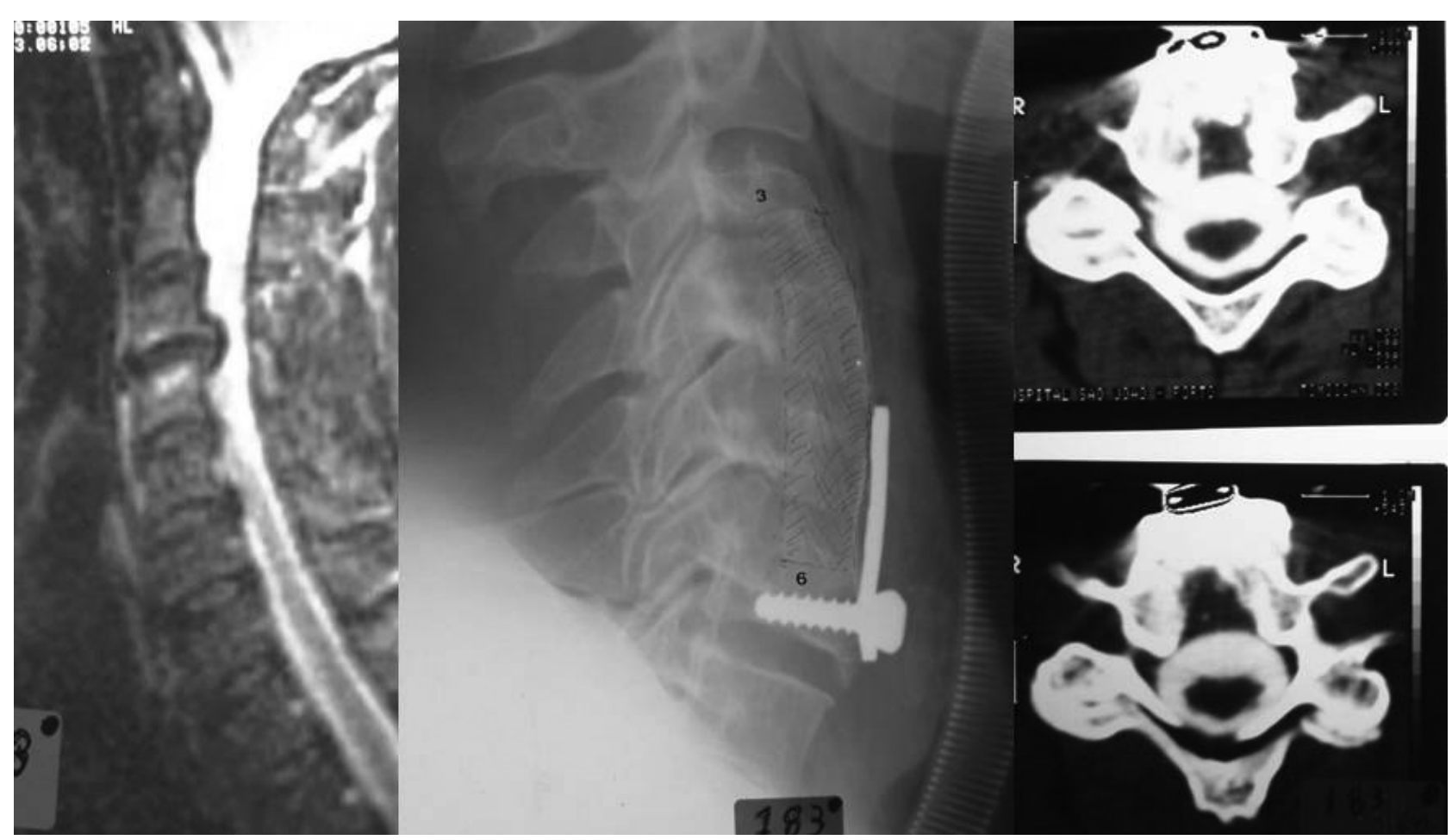

Figura 2

Técnica de Sénegas ${ }^{8}$ para compressões em dois ou mais níveis

A análise de correlação permitiu identificar uma forte correlação entre a idade e o número de níveis operados $(\mathrm{r}=0,391 ; \mathrm{p}=0,01), \mathrm{a}$ idade e o estado neurológico inicial $(\mathrm{r}=0,238 ; \mathrm{p}=0,05)$, o estado neurológico inicial e o número de níveis operados $(\mathrm{r}=0,251 ; \mathrm{p}=0,05)$ e o sexo e o número de níveis operados, sendo que as mulheres apresentaram um maior número de níveis operados $(\mathrm{r}=0,208 ; \mathrm{p}=0,05)$. Verificou-se que indivíduos mais jovens apresentaram maior degradação neurológica entre o primeiro ano após operação e a última revisão $(\mathrm{r}=0,25 ; \mathrm{p}=0,05)$ e uma forte correlação entre a recuperação neurológica ao final de um ano e a recuperação final $(\mathrm{r}=0,838 ; \mathrm{p}=0,01)$.

Segundo os processos, foi observada consolidação do enxerto em todos os casos. Contudo, em quatro pacientes verificou-se colapso do enxerto ósseo, mas em todos houve melhoria clínica ao final do primeiro ano. Três desses casos tinham sido operados em quatro níveis e outro em três níveis. Um desses não se apresentou à revisão final, houve uma degradação de um ponto na escala de Nurick (de dois para três) num caso e manutenção do resultado nos outros dois. Três doentes $(4,1 \%)$ foram reintervencionados por descompensação do nível adjacente, quatro, oito e dez anos após a cirurgia inicial.

\section{DISCUSSÃO}

Esta série é uma revisão retrospectiva dos resultados a longo prazo da descompressão e artrodese anteriores, realizadas numa única instituição, por dois cirurgiões, que utilizaram técnicas reprodutíveis em doentes com mielopatia cervical espondilótica. Como outros investigadores, procuramos identificar os fatores preditivos clínicos e radiográficos da gravidade da mielopatia pré e pós-operatória $^{9-12}$. A significativa melhoria do estado neurológico, quer no primeiro ano após a operação, quer à data da revisão final, é a principal conclusão dos nossos resultados. Verificou-se igualmente uma forte associação entre a recuperação neurológica inicial e a melhoria clínica final obtida $(\mathrm{p}=0,01)$.

Diversos autores mostram resultados satisfatórios com a cirurgia de descompressão e artrodese anterior ${ }^{2,13-15}$. Emery et al. ${ }^{13}$ relataram 106 doentes com mielopatia cervical, que foram sujeitos a corporectomia ou discectomia anterior e artrodese, seguidos entre 2 e 17 anos; $77 \%$ dos doentes apresentavam alterações pré-operatórias da função motora, relacionada a marcha e/ou equilíbrio. Desses pacientes, cerca de $46 \%$ obtiveram uma recuperação completa da função motora e $40 \%$ obtiveram uma melhoria na capacidade de marcha com melhoria do seu estado de Nurick de 2,4 para 1,2. Okada et al. relataram 37 doentes que realizaram uma artrodese cervical após descompressão de 1 a 4 níveis. O tempo médio de seguimento foi de quatro anos. Todos os pacientes apresentavam alterações motoras no pré-operatório, sendo que somente $46 \%$ desses doentes apresentavam-na no pós-operatório ${ }^{16}$. Muito embora não haja nenhum estudo prospectivo e randomizado, esses resultados confirmam o benefício da cirurgia face à história natural dessa doença ${ }^{17}$.

Não existem na literatura diferenças significativas em termos de resultado neurológico quando comparados os resultados de seguimento de abordagens anteriores versus 
abordagens posteriores ${ }^{18,19}$. A utilização de uma via de abordagem anterior permite uma boa visualização das anomalias que estão localizadas ventralmente, evitando a necessidade de abordar em torno da medula. Excelentes resultados têm sido apresentados com a utilização dessa via, fazendo dela a primeira opção no tratamento da maior parte dos quadros de mielopatia cervical. A corporectomia deve ser considerada em doentes com estenoses em que o diâmetro anteroposterior do canal vertebral é menor que $13 \mathrm{~mm}$, doentes com osteófitos posteriores adjacentes à placa vertebral e que resultem em compressão medular, doentes com fragmentos discais livres e migrados posteriormente no canal ou como alternativa a artrodeses intersomáticas em múltiplos níveis, diminuindo, dessa forma, as superfícies de artrodese. Também a correção de uma deformidade fixa em cifose pode ser obtida por meio de uma corporectomia anterior, em comparação com múltiplas discectomias e artrodeses ${ }^{20,21}$.

Para doentes com envolvimento de um a dois níveis, a maior parte dos cirurgiões prefere a utilização de um procedimento anterior ${ }^{21,22}$. Yonenobu et al., num estudo com doentes com mielopatia cervical, reportaram que nos comprometidos de um a dois níveis eram tratados por meio de discectomia anterior ou corporectomia, ao passo que descompressões de quatro ou mais níveis eram tratadas por meio de laminectomia. Para doentes com envolvimento de três níveis, qualquer uma das três opções era considerada válida $^{21}$. Doentes submetidos a corporectomia mantiveram o seu estado de recuperação neurológica de uma forma mais estável e durante mais tempo, em comparação com os doentes sujeitos a outros tipos de procedimentos descompressivos. Os autores recomendam a realização de procedimentos de descompressão anteriores, principalmente a corporectomia, em casos de comprometimento até três níveis. Deve ser considerado o risco de cifose pós-operatória com o número crescente de níveis descomprimidos. Pelo contrário, Ikenaga et al., em 2008, numa série de 31 doentes sujeitos a descompressão de quatro ou mais níveis por via anterior, apresentam excelentes resultados clínicos e radiológicos com seguimento mínimo de $10 \operatorname{anos}^{23}$. Na nossa série, dos quatro casos em que se observou colapso do enxerto, três correspondiam a descompressões a quatro níveis, tendo um deles sofrido um agravamento do estado neurológico no seguimento final. A opção para descompressões de quatro ou mais níveis é decidida caso a caso.

O colapso do enxerto e consequente cifose poderão ser evitados com a utilização de placas cervicais ${ }^{24,25}$. As placas anteriores aumentam as taxas de fusão, reduzem o tempo, alteram o tipo de imobilização pós-operatória e diminuem o número de complicações associadas ao enxerto, bem como as taxas de cifose pós-operatória, em especial em doentes submetidos a descompressão e fusão de mais de dois níveis, ajudando a manter ou até a melhorar a lordose cervical fisiológica ${ }^{26-29}$. Num estudo em que foi realizada análise computorizada de uma radiografia lateral da coluna cervical em 51 doentes submetidos a descompressão e artrodese cervical de um ou mais níveis, verificou-se um benefício significativo $(\mathrm{p}=0,0001)$ da utilização de placa anterior na manutenção do alinhamento sagital pós-operatório ${ }^{30}$. À data da realização das cirurgias avaliadas neste estudo, não utilizávamos por rotina placas cervicais nas descompressões a um nível, ao passo que, em descompressões múltiplas, era utilizada uma placa em batente fixada na vértebra inferior. Foi obtida uma taxa de fusão de $100 \%$. Nas séries de Ikenaga et al. ${ }^{23} \mathrm{e}$ Bernard e Whitecloud ${ }^{31}$, são obtidas taxas de consolidação semelhantes em doentes também submetidos a corporectomia e artrodese anterior sem a utilização de placa. Contudo, face às complicações associadas ao enxerto, à possibilidade de reduzir o tempo de imobilização e à evidência descrita na literatura, atualmente incluímos o uso de placas na maioria dos nossos procedimentos descompressivos anteriores.

As taxas de morbilidade e mortalidade nesse grupo de doentes não são desprezíveis, como mostram as cinco mortes ocorridas no pós-operatório imediato e precoce na nossa série. A condição médica do doente deve ser um critério de decisão importante na consideração do tratamento cirúrgico, dado que o resultado final é influenciado pelas morbilidades médicas subjacentes, em especial nos idosos. Ainda que com monitorização e vigilância clínica eficaz no período pré e intraoperatório, a cirurgia pode resultar num agravamento clínico dos doentes ${ }^{32,33}$. Deterioração funcional no pós-operatório pode ocorrer por consequência de enfarte cerebral ou descompensação da diabetes mellitus. Alterações associadas à idade no sistema nervoso central, osteoartrose coexistente nos membros inferiores, estenose lombar e neuropatias periféricas podem impedir ou prejudicar uma recuperação neurológica mais franca após a descompressão e artrodese cervical ${ }^{33}$.

Diversos estudos mostram que doentes com mais de 75 anos de idade apresentam maiores comorbilidades e, em geral, não obtêm o mesmo grau de recuperação pós-operatória que os doentes mais novos ${ }^{33}$. Alterações desmielinizantes da medula, estenose vertebral lombar, coxartrose, insuficiência cardíaca podem contribuir para um quadro de disfunção motora, diminuição da capacidade de marcha e agravamento do estádio de Nurick, juntamente com a mielopatia cervical, bem como diminuir os resultados que seriam de se esperar do tratamento cirúrgico ${ }^{32,34}$.

Pelo contrário, outros estudos preconizam que a descompressão cirúrgica deve ser realizada mesmo em doente com mais de 80 anos, desde que o tempo que decorra a partir do início do quadro clínico seja inferior a três anos, a incapacidade de marcha seja inferior a três meses e o doente possa suportar uma cirurgia ${ }^{35}$. Também na nossa série registamos uma melhoria significativa nos doentes operados independentemente da idade. A maioria dos doentes melhorou um ou dois graus na escala de Nurick. Contudo, tal como é referido na maioria das séries ${ }^{13,23}$, doentes mais idosos apresentavam um estado neurológico inicial mais avançado, pelo que, apesar de uma recuperação 
semelhante, o estado final é pior quando comparado com doentes mais novos.

As intervenções cirúrgicas que são efetuadas na fase inicial e em estádio mais precoce da doença também aparentam ter melhores resultados em termos de melhoria neurológica dos doentes ${ }^{35-38}$. Num estudo prospectivo de 146 doentes com mielopatia cervical espondilótica, Suri et al. declaram que doentes com menos de um ano de duração dos sintomas mostraram recuperação da função motora significativamente melhor em relação a doentes que foram intervencionados após mais tempo de evolução do quadro clínico $(p<0,05)^{39}$. Tanaka et al. mostraram, num estudo com 47 doentes que tinham mais de 65 anos de idade, que o tempo de desenvolvimento pré-operatório do quadro clínico influencia claramente a recuperação funcional dos doentes após intervenção cirúrgica ${ }^{35}$.

Apesar do maior número de níveis intervencionados nos doentes do sexo feminino, não se verificou uma diferença na recuperação neurológica entre os dois sexos, contrariamente ao sugerido por Emery et al. ${ }^{13}$

Poucos são os estudos que avaliam uma série tão extensa e com tão grande tempo de seguimento, embora uma tendência geral seja observada em termos do padrão de recuperação após cirurgia para tratamento da mielopatia cervical: a recuperação que é obtida imediatamente após a cirurgia é seguida por uma melhoria nos primeiros anos e depois por um novo agravamento progressivo. Wada et al., num estudo retrospectivo de 23 doentes com mielopatia cervical espondilótica, tratados por corporectomia anterior e artrodese cervical, relataram uma melhoria no JOA (Japanese Orthopaedic Score) de 7,9 pré-operatóriamente para 13,3 após 1 ano de cirurgia e 13,9 após 5 anos; 15 anos após a cirurgia, o score observado era de $13,4^{15}$. Ikenaga et al. relataram resultados clínicos estáveis para mais de dez anos, desde que haja remoção completa das causas possíveis de mielopatia ${ }^{23}$.

A causa mais frequente de sintomas após procedimentos por via anterior, referida na literatura, é a degeneração do segmento adjacente, tal como também se verificou na nossa série. Numa análise de 75\% de doentes, 21 anos após fusão cervical, Hilibrand et al. relataram que, a cada ano, 2,9\% desenvolviam clínica compatível com degeneração do nível adjacente ${ }^{40}$. A análise de sobrevivência de Kaplan-Meier indicou que o risco de desenvolvimento de doença no nível adjacente era de 25,6\% em 10 anos após a cirurgia cervical. Os níveis C5-C5 e C6-C7 foram aqueles que mais frequentemente apresentaram alterações degenerativas enquanto discos adjacentes, mas também são aqueles com maior predisposição a apresentar naturalmente alterações degenerativas. Alguns autores chegam mesmo a afirmar que o fenómeno de agravamento neurológico não se encontra relacionado com a degeneração nos discos adjacentes após a cirurgia, mas sim que decorre de um processo de envelhecimento e degeneração do esqueleto axial, processo natural e independente da existência ou não de cirurgia prévia. Alguns estudos de biomecânica cervical apoiam isso, mesmo ao mostrarem que a artrodese cervical no contexto de discectomia ou corporectomia não predispõe a degeneração do nível adjacente ${ }^{41}$. Wada et al. estudaram a degeneração dos níveis adjacentes em 23 doentes após artrodese anterior e reportaram que cerca de $54 \%$ apresentavam desenvolvimento de osteófitos e estreitamento do espaço intervertebral nos níveis adjacentes. No entanto, só um desses necessitou de cirurgia de recidiva de mielopatia cervical num período de $15 \operatorname{anos}^{15}$. Na nossa série, observou-se uma pequena, mas estatisticamente significativa, degradação clínica entre o primeiro e o último tempo de seguimento pós-operatório, que, no entanto, só obrigou a reintervenção em três casos.

Outras causas de agravamento neurológico a longo prazo podem incluir descompressão insuficiente na cirurgia inicial, pseudartrose, colapso ou deslocamento do enxerto, desenvolvimento de novos osteófitos ou invaginação do ligamento amarelo. Contrariamente ao observado na maioria das séries, verificamos um maior agravamento a longo prazo nos doentes mais jovens. O maior número de níveis intervencionados em doentes mais idosos poderá ter correspondido à utilização de uma cirurgia menos agressiva nos pacientes mais jovens, possibilitando a degradação progressiva nos segmentos adjacentes à área de fusão. Hilibrand et al. relatam que o desenvolvimento desse quadro clínico pode ser ainda mais precoce se o doente previamente à fusão já apresentar sinais de degeneração nos discos adjacentes, pelo que se devem incluir na descompressão e fusão inicial todos os níveis claramente degenerados ${ }^{40,42}$.

\section{CONCLUSÃO}

Nosso estudo, de longo prazo, dos resultados da descompressão anterior e artrodese no tratamento da mielopatia cervical espondilótica demonstrou altas taxas de recuperação da função neurológica na maioria dos doentes.

O fator preditivo mais importante da recuperação obtida a longo prazo é a melhoria conseguida no pós-operatório inicial.

Embora haja uma pequena degradação clínica ao longo do tempo, relacionada, na maioria dos casos, com degenerescência do segmento adjacente, poucos são os doentes que necessitam de reintervenção.

A complicação mais frequentemente observada, apesar de não ter tido repercussão clínica, foi o colapso do enxerto, pelo que para intervenções a três ou mais níveis se deve considerar a possibilidade de utilização de placa ou via posterior. 


\section{REFERÊNCIAS}

1. Rao R. Neck pain, cervical radiculopathy, and cervical myelopathy: pathophysiology, natural history, and clinical evaluation. J Bone Joint Surg Am. 2002;84-A(10): 1872-81.

2. Sampath P, Bendebba M, Davis JD, Ducker TB. Outcome of patients treated for cervical myelopathy. A prospective, multicenter study with independent clinical review. Spine (Phila Pa 1976). 2000;25(6):670-6.

3. Hirabayashi K, Miyakawa J, Satomi K, Maruyama T, Wakano K. Operative results and postoperative progression of ossification among patients with ossification of cervical posterior longitudinal ligament. Spine (Phila Pa 1976). 1981;6(4):354-64.

4. Tsuji H. Laminoplasty for patients with compressive myelopathy due to so-called spinal canal stenosis in cervical and thoracic regions. Spine (Phila Pa 1976). 1982;7(1):28-34.

5. Robinson RA, Smith GW. Anterolateral cervical disc removal and interbody fusion for cervical disc syndrome. Bull John Hopkins Hosp. 1955;96:223-4.

6. Cloward RB. The anterior approach for removal of ruptured cervical disks. J Neurosurg. 1958;15(6):602-17.

7. Simmons EH, Bhalla SK. Anterior cervical discectomy and fusion. A clinical and biomechanical study with eight-year follow-up. J Bone Joint Surg Br. 1969;51(2):225-37.

8. Sénegas V, Guérin V. Techniques de decompression medulaire anterieure dans les stenose canallaires étendues. Rev Chir Orthop. 1975;61 Suppl 2:219-33.

9. Fujiwara K, Yonenobu K, Ebara S, Yamashita K, Ono K. The prognosis of surgery for cervical compression myelopathy. An analysis of the factors involved. J Bone Joint Surg Br. 1989;71(3):393-8.

10.Hayashi H, Okada K, Hashimoto J, Tada K, Ueno R. Cervical spondylotic myelopathy in the aged patient. A radiographic evaluation of the aging changes in the cervical spine and etiologic factors of myelopathy. Spine (Phila Pa 1976). 1988;13(6):618-25.
11. Ogino H, Tada K, Okada K, Yonenobu $\mathrm{K}$, Yamamoto T, Ono K, et al. Canal diameter, anteroposterior compression ratio, and spondylotic myelopathy of the cervical spine. Spine (Phila $\mathrm{Pa}$ 1976). 1983;8(1):1-15.

12.Wolf BS, Khilnani M, Malis L. The sagittal diameter of the bony cervical spinal canal and its significance in cervical spondylosis. J M Sinai Hosp N Y. 1956;23(3):283-92.

13.Emery SE, Bohlman HH, Bolesta MJ, Jones PK. Anterior cervical decompression and arthrodesis for the treatment of cervical spondylotic myelopathy. Two to seventeen-year follow-up. J Bone Joint Surg Am. 1998;80(7):941-51.

14.Kadanka Z, Mares M, Bednarík J, Smrcka V, Krbec M, Chaloupka R, et al. Predictive factors for spondylotic cervical myelopathy treated conservatively or surgically. Eur J Neurol. 2005;12(1):55-63.

15. Wada E, Suzuki S, Kanazawa A, Matsuoka T, Miyamoto S, Yonenobu K. Subtotal corpectomy versus laminoplasty for multilevel cervical spondylotic myelopathy: a long-term follow-up study over 10 years. Spine (Phila Pa 1976). 2001;26(13):1443-8.

16.Okada K, Shirasaki N, Hayashi H, Oka S, Hosoya T. Treatment of cervical spondylotic myelopathy by enlargement of the spinal canal anteriorly, followed by arthrodesis. J Bone Joint Surg Am. 1991;73(3): 352-64.

17.Clarke E, Robinson PK. Cervical myelopathy: a complication of cervical spondylosis. Brain. 1956;79(3):483510.

18.Sakaura H, Hosono N, Mukai Y, Ishii T, Iwasaki M, Yoshikawa H. Long-term outcome of laminoplasty for cervical myelopathy due to disc herniation: a comparative study of laminoplasty and anterior spinal fusion. Spine (Phila Pa 1976). 2005;30(7):756-9.

19.Yonenobu K, Hosono N, Iwasaki M, Asano M, Ono K. Laminoplasty versus subtotal corpectomy. A comparative study of results in multisegmental cervical spondylotic myelopathy. Spine (Phila Pa 1976). 1992;17(11):1281-4.
20.Kadoya S, Nakamura T, Kwak R. A microsurgical anterior osteophytectomy for cervical spondylotic myelopathy. Spine (Phila Pa 1976). 1984;9(5):437-41.

21. Yonenobu K, Fuji T, Ono K, Okada K, Yamamoto T, Harada N. Choice of surgical treatment for multisegmental cervical spondylotic myelopathy. Spine (Phila Pa 1976). 1985;10(8):710-6.

22.Kawakami M, Tamaki T, Iwasaki H, Yoshida M, Ando M, Yamada H. A comparative study of surgical approaches for cervical compressive myelopathy. Clin Orthop Relat Res. 2000;(381):129-36.

23.Ikenaga M, Shikata J, Tanaka C. Longterm results over 10 years of anterior corpectomy and fusion for multilevel cervical myelopathy. Spine (Phila Pa 1976). 2006;31(14):1568-74.

24. Vaccaro AR, Falatyn SP, Scuderi GJ, Eismont FJ, McGuire RA, Singh K, et al. Early failure of long segment anterior cervical plate fixation. J Spinal Disord. 1998;11(5):410-5.

25.Sasso RC, Ruggiero RA Jr, Reilly TM, Hall PV. Early reconstruction failures after multilevel cervical corpectomy. Spine (Phila Pa 1976). 2003;28(2):140-2.

26.Connolly PJ, Esses SI, Kostuik JP. Anterior cervical fusion: outcome analysis of patients fused with and without anterior cervical plates. J Spinal Disord. 1996; 9(3):202-6.

27.Kaiser MG, Haid RW Jr, Subach BR, Barnes B, Rodts GE Jr. Anterior cervical plating enhances arthrodesis after discectomy and fusion with cortical allograft. Neurosurgery. 2002;50(2):229-38.

28.Wang JC, McDonough PW, Endow KK, Delamarter RB. Increased fusion rates with cervical plating for twolevel anterior cervical discectomy and fusion. Spine (Phila Pa 1976). 2000;25(1):41-5.

29.Wang JC, McDonough PW, Endow K, Kanim LE, Delamarter RB. The effect of cervical plating on singlelevel anterior cervical discectomy and fusion. J Spinal Disord. 1999;12(6):467-71.

30.Herrmann AM, Geisler FH. Geometric results of anterior cervical plate stabilization in degenerative disease. Spine (Phila Pa 1976). 2004;29(11):1226-34. 
31.Bernard TN Jr, Whitecloud TS 3rd. Cervical spondylotic mielopathy and myeloradiculopathy. Anterior decompression and stabilization with autogenous fibula strut graft. Clin Orthop Relat Res. 1987;(221):149-60.

32.Kawaguchi Y, Kanamori M, Ishihara H, Ohmori K, Abe Y, Kimura T. Pathomechanism of myelopathy and surgical results of laminoplasty in elderly patients with cervical spondylosis. Spine (Phila Pa 1976). 2003;28(19):2209-14.

33. Matsuda Y, Shibata T, Oki S, Kawatani Y, Mashima N, Oishi H. Outcomes of surgical treatment for cervical myelopathy in patients more than 75 years of age. Spine (Phila Pa 1976). 1999;24(6):529-34.

34.Nurick S. The pathogenesis of the spinal cord disorder associated with cervical spondylosis. Brain. 1972;95(1):87-100.

35.Tanaka J, Seki N, Tokimura F, Doi K, Inoue S. Operative results of canalexpansive laminoplasty for cervical spondylotic myelopathy in elderly patients. Spine (Phila Pa 1976). 1999;24(22):2308-12.
36.Lee TT, Manzano GR, Green BA. Modified open-door cervical expansive laminoplasty for spondylotic myelopathy: operative technique, outcome, and predictors for gait improvement. J Neurosurg. 1997;86(1):64-8.

37.Hukuda S, Mochizuki T, Ogata M, Shichikawa K, Shimomura Y. Operations for cervical spondylotic myelopathy. A comparison of the results of anterior and posterior procedures. J Bone Joint Surg Br. 1985;67(4):609-15.

38.Chiles BW 3rd, Leonard MA, Choudhri HF, Cooper PR. Cervical spondylotic myelopathy: patterns of neurological deficit and recovery after anterior cervical decompression. Neurosurgery. 1999;44(4):762-70.

39. Suri A, Chabbra RP, Mehta VS, Gaikwad S, Pandey RM. Effect of intramedullary signal changes on the surgical outcome of patients with cervical spondylotic myelopathy. Spine J. 2003;3(1):33-45.

40.Hilibrand AS, Carlson GD, Palumbo MA, Jones PK, Bohlman HH. Radiculopathy and myelopathy at segments adjacent to the site of a previous anterior cervical arthrodesis. J Bone Joint Surg Am. 1999;81(4):519-28.
41.Rao RD, Wang M, McGrady LM, Perlewitz TJ, David KS. Does anterior plating of the cervical spine predispose to adjacent segment changes? Spine (Phila Pa 1976). 2005;30(24):2788-93.

42.Hilibrand A, Berta S, Daffner S. The impact of anterior cervical fusion upon overall range of motion and neck flexibility. In: Proceedings of the Annual Meeting of the North American Spine Society; 2003 Oct 21-25; San Diego, CA, USA; 2003.

\section{Correspondência}

Manuel Ribeiro da Silva

Serviço de Ortopedia do Hospital de São João

Alameda Professor Hernani Monteiro, 4.200

Porto - Portugal

Tel.: (+351) 225512100

E-mail:manuelrisilva@gmail.com 